\title{
Apply Matlab in Thingspeak Server to build the system measure and analyze data using IoT Gateway technology
}

\author{
Chi Van Dang ${ }^{*}$, Khoat Duc Nguyen, Hieu Dao, Luc The Nguyen
}

Faculty of Electromechanics, Hanoi University of Mining and Geology, Vietnam

ARTICLE INFO ABSTRACT

Article history:

Received 22nd June 2020

Revised 05 ${ }^{\text {th }}$ Sept. 2020

Accepted 31 ${ }^{\text {st }}$ Oct. 2020

Keywords:

IoT Gateway, Web Server, LoRa Gateway LG01-N, MATLAB® in Thingspeak, MQTT Protocol.
ThingSpeak is an open Internet of Things (IoT) platform with MATLAB® analytics that enables the collection and storage of sensor data in the cloud and development of IoT applications. The ThingSpeak IoT platform provides applications that allow data analysis and visualization in MATLAB. With MATLAB $®$ analysis in ThingSpeak, MATLAB code can be executed to perform preprocessing, visualization, filtering, data analysis, and for object modeling applications. This paper presents researches on Matlab application in Thingspeak Server to build data measurement and analysis system using IoT LoRa Gateway technology. The research contents include suggestions on device configuration for the system, programming the Arduino board and LoRa Shield to collect measurement data from sensor nodes and communicate by LoRa waves to the LoRa Gateway. The LoRa Gateway will send data to Web Server based on Thingspeak's Cloud Service platform using MQTT (Message Queing Telemetry Transport). Thingspeak's Matlab interface will display online and store values from the sensor nodes. The system is integrated and tested on temperature and humidity monitoring model, evaluated for the results with the required accuracy. The research results allow the deployment of IoT Gateway system in practice for online measurement, analysis and data processing applications that require the use of algorithms and code generation in Matlab using Web Server.

Copyright (C) 2020 Hanoi University of Mining and Geology. All rights reserved.

${ }^{*}$ Corresponding author E - mail: dangvanchi@humg.edu.vn DOI: 10.46326/JMES.2020.61(5).10 


\title{
Tạp chí Khoa học Kỹ thuật Mỏ - Địa chất
}

Trang điện tử: http://tapchi.humg.edu.vn

\section{Ứng dụng Matlab trong Thingspeak Server để xây dựng hệ thống đo lường và phân tích dữ liệu từ xa bằng công nghệ IoT Gateway}

\author{
Đặng Văn Chí ${ }^{*}$, Nguyễn Đức Khoát, Đào Hiếu, Nguyễn Thế Lực
}

Khoa Cơ Điện, Trường Đại học Mỏ - Địa chất, Việt Nam

THÔNG TIN BÀI BÁO T TÓM TẮT

Quá trình:

Nhận bài 22/6/2020

Sưa xong 05/9/2020

Chấp nhận đăng 31/10/2020

Tù̀ khóa:

IoT Gateway, Web Server, LoRa Gateway LG01-N, MATLABß in Thingspeak, MQTT Protocol.
ThingSpeak là một nền tảng Internet of Things (IoT) mở với các phân tích MATLAB® cho phép thu thập và lưu dữ liệu cảm biến trên đám mây và phát triển các úng dung IoT. Nền tảng ThingSpeak IoT cung cấp các ứng dung cho phép phân tích và trực quan hóa dũ liệu trong MATLAB. Có thể viết và thưc thi mã MATLAB® bên trong ThingSpeak đê thưc hiên quá trình tiền xử lý, trực quan hóa, lọc, phân tích dũ liệu và cho các ứng dụng mô hình hóa đối tượng. Bài báo này trình bày nhũng nghiên cúu bước đầu trong việc úng dưng Matlab trong Thingspeak Server để xây dựng hệ thống đo lường tù xa bằng công nghê IoT LoRa Gateway. Nội dung nighiên cứu bao gồm đề xuất cấu hình thiết bi cho hê thống, lập trình code cho board Arduino và LoRa Shield để thu thập dữ liệu đo từ các sensor node và truyền thông không dây bằng sóng LoRa đến LoRa Gateway. LoRa Gateway sẽ gửi dũ liệu lên Web Server dựa trên nền tảng Cloud Service của Thingspeak bằng giao thúc MQTT (Message Queing Telemetry Transport). Giao diện được thiết lập trên Matlab của Thingspeak sẽ hiển thi trực tuyến và lưu giá tri đoo tù các sensor node. Hệ thống được tích hợp và chạy thư trên mô hình giám sát nhiệt độ và độ ẩm môi trường, bước đầu đánh giá cho kết quả với độ chính xác theo yêu cầu. Kết quả nghiên cúu cho phép triển khai hệ thống IoT Gateway trong thực tế với các ứng dụng đo lường, phân tích và xử lý dữ liẹu trực tuyến với yêu cầu sử dụng thuật toán và tạo mã trong Matlab bằng Web Server.

C 2020 Trường Đại học Mỏ - Địa chất. Tất cả các quyền được bảo đảm.

\section{Mở đầu}

Công nghệ IoT đang trở thành xu hướng phát triển mới trên thế giới, IoT được định nghĩa là những đối tượng (Things) có khả năng kết nối

*Tác giả liên hệ

E - mail: dangvanchi@humg.edu.vn

DOI: 10.46326/JMES.2020.61(5).10 mạng Internet và tác động qua lại giữa các dịch vụ web. Nó cho phép nhiều đối tượng được nhúng với các giao diện truyền thông có dây hoặc không dây để tự động kết nối và tương tác với nhau (Nguyễn Đức Khoát, 2014). IoT là một hệ thống liên kết các hệ điều khiển nhúng, các cảm biến, phần mềm và mạng (Cao Hoàng Tiến, 2015; (Nguyễn Văn Phong 2018). 
ThingSpeak là một dịch vụ nền tảng phân tích IoT cho phép tổng hợp và phân tích các trường dữ liệu trực tiếp trên đám mây. Có thể gửi dữ liệu tới ThingSpeak từ các Device và các Sensor node,... tạo các Trend dữ liệu trực tiếp và gửi thông báo bằng các dịch vụ web. Với các phân tích bằng công cụ MATLABß bên trong ThingSpeak, có thể viết và thực thi mã MATLAB để thực hiện quá trình tiền xử lý, phân tích dữ liệu, hiển thị đồ thị giao diện người dùng, liên kết với các chương trình sử dụng nhiều ngôn ngữ khác nhau,... Đồng thời, ThingSpeak cho phép các kỹ sư và nhà khoa học xây dựng các hệ thống IoT mà không cần thiết lập máy chủ hoặc phát triển phần mềm web (Vũ Thị Quyên và nnk., 2018; Phạm Quang Huy, Nguyễn Trọng Hiếu, 2016).

Hiện nay trong nước cũng đã có nhiều công trình được các nhà khoa học nghiên cứu và từng bước triển khai các giải pháp đo lường giám sát từ xa như đề tài: "Phương pháp giám sát và điều khiển các thông số môi trường trên nền tảng điện toán đám mây qua mạng truyền thông không dây WIMAX", của tác giả Phạm Ngọc Minh \& nnk., (2015). Đề tài nghiên cứu cấp nhà nước "Ảnh hưởng của Enso đến các cực trị và lượng mưa ở Việt Nam và khả năng dự báo", tác giả Nguyễn Đức Ngũ̃, (2012), và đề tài thuộc dự án PAM Air "Nghiên cứu hệ thống giám sát chất lượng không khí cho các mỏ than lộ thiên Quảng Ninh" được thực hiện với sự hợp tác giữa Đại học Đông Á (Hàn Quốc) và Đại học Mỏ - Địa chất, thực hiện năm 2018-2020 (Dự án PAM Air, 2018-2020).

Trong nghiên cứu này các tác giả đề xuất giải pháp ứng dụng phân tích MATLAB được tích hợp nhúng bên trong Thingspeak ${ }^{\mathrm{TM}}$ để lọc, xử lý và phân tích dữ liệu. Việc sử dụng các công cụ của Matlab trong Thingspeak Sever đặc biệt thích hợp cho các bài toán IoT có yêu cầu xử lý các thuật toán phức tạp, phù hợp cho các bài toán xử lý online trong các hệ thống điều khiển thông minh ứng dụng các thuật toán AI, Fuzzy Logic, Neural Network và dữ liệu lớn,...

\section{Phương pháp nghiên cứu}

\subsection{Xây dựng thiết kế phần cứng}

\subsubsection{Xây dựng mô hình nguyên lý hệ thống}

LoRa (Long Range Radio) được nghiên cứu và phát triển bởi Cycleo, công nghệ này có thể truyền dữ liệu với khoảng cách hàng km mà không cần các mạch khuếch đại công suất, giúp tiết kiệm năng lượng tiêu thụ khi truyền và nhận dữ liệu (Emara, và nnk., 2009). Do đó, LoRa được áp dụng rộng rãi trong các ứng dụng thu thập dữ liệu như sensor network. Trong đó, các sensor node có thể gưii giá trị đo về trung tâm cách xa hàng $\mathrm{km}$ và có thể hoạt động với pin trong thời gian dài (Pushpa Methekar, Girish Talmale, 2015); Nasiruddin, Rashida Shujaee, 2017) (Hình 1).

LoRa sử dụng kỹ thuật điều chế Chirp Spread Spectrum với nguyên lý "băm" dữ liệu bằng các xung cao tần để tạo ra tín hiệu có dãy tần số cao hơn tần số của dữ liệu gốc (gọi là chipped). Sau đó, tín hiệu cao tần này tiếp tục được mã hoá theo các chuỗi chirp signal (là các tín hiệu hình sin có tần số thay đổi theo thời gian). Có 2 loại chirp signal là up-chirp có tần số tăng theo thời gian và downchirp có tần số giảm theo thời gian. Việc mã hoá theo nguyên tắc bit 1 sẽ sử dụng up-chirp và bit 0 sẽ sử dụng down-chirp trước khi truyền ra anten để gửi đi.

Theo mô hình trên, hệ thống được thiết lập với các thiết bị hoạt động như sau:

- Thiết bị hiện trường: là hệ thống các cảm biến dữ liệu sensor node đều được gắn các module LoRa.

- Các dữ liệu cảm biến truyền về bộ LoRa Gateway đặt tại trung tâm khu vực địa lý đó. Thông qua Gateway, dữ liệu có thể truyền về trung tâm (không giới hạn khoảng cách địa lý) thông qua mạng GPRS/3G hoặc thông qua Internet.

- Giao diện Matlab được thiết lập trên Thingspeak sẽ hiển thị online và lưu trữ giá trị đo từ các sensor node.

\subsubsection{Thiết bị LoRa Gateway LG01-N.}

LG01-N là thiết bị LoRa Gateway mã nguồn mở. Cho phép kết nối mạng không dây LoRa với mạng IP thông qua wifi ethernet, mạng di động 3G/4G. LG01-N chạy trên hệ điều hành Linux mã nguồn mở, có cổng USB host được sử dụng để kết nối các module di động rất linh hoạt và kết nối mạng LoRa với các loại mạng khác nhau, phù hợp với yêu cầu của người sử dụng (Qian và nnk., 2010) (Hình 2).

Thông số kỹ thuật LG01-N:

- Bộ vi xử lý: 400Mhz, AR9331.

- Bộ nhớ: 16MB; RAM 64MB.

- Giải tần số:

+ Band 1 (HF): 862 1020 Mhz. 


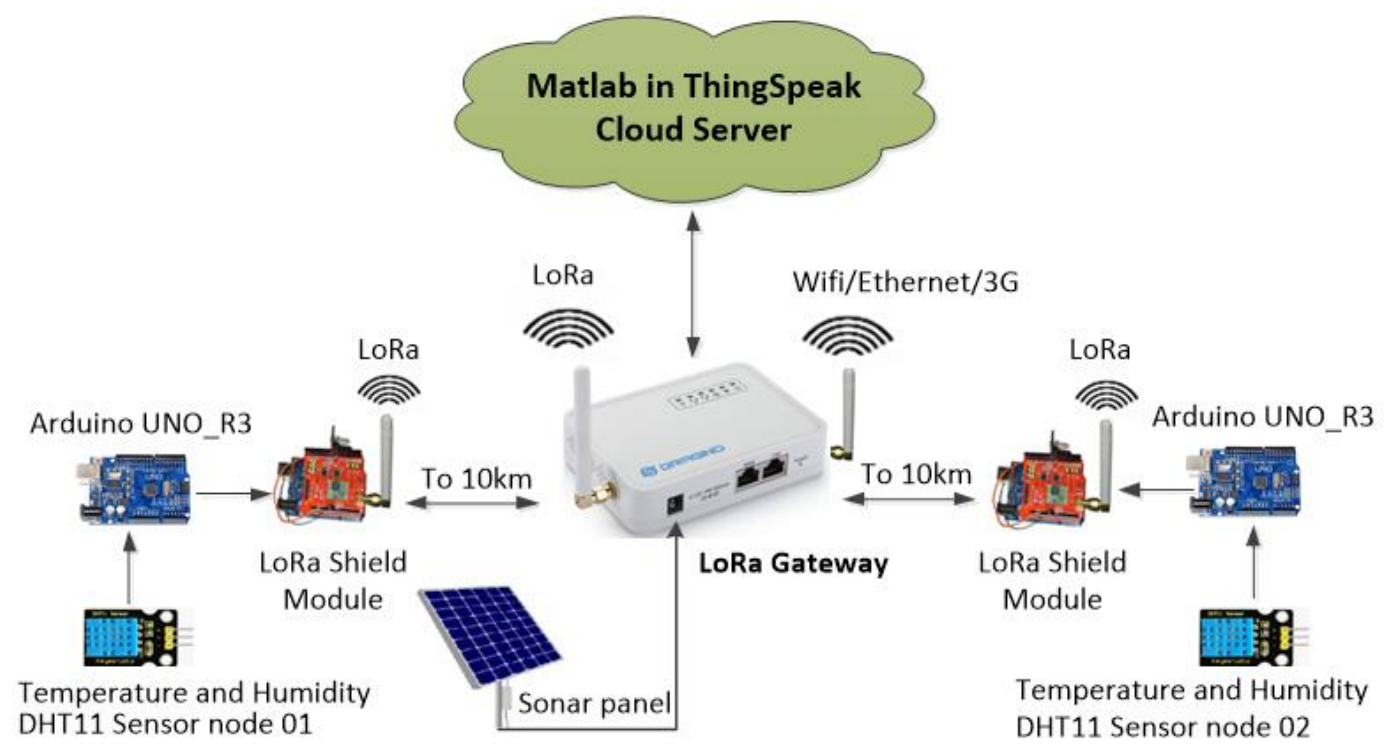

Hình 1. Sơ đồ nguyên lý hệ thống IoT Gateway.

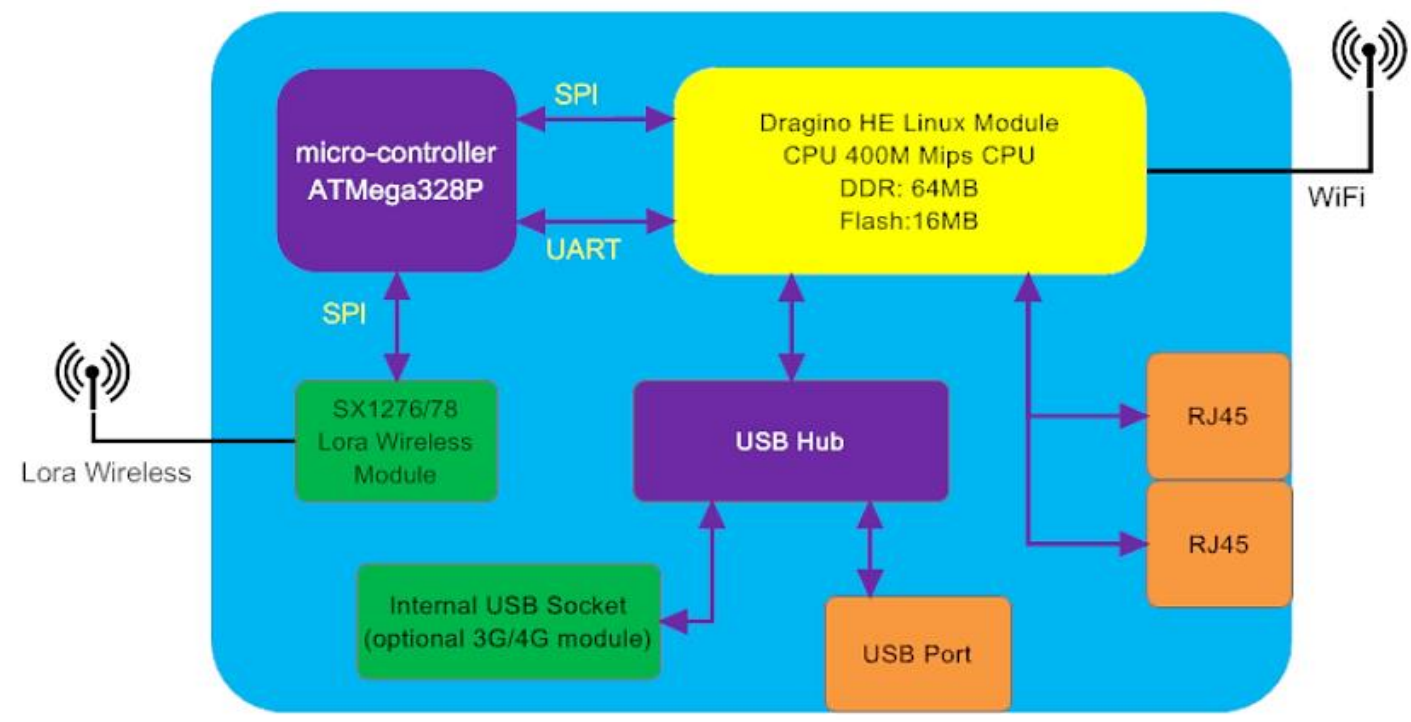

Hình 2. Sơ đồ nguyên lý LoRa IoT Gateway LG01-N

+ Band 2 (LF): $410 \sim 528$ Mhz.

- Mạng 4G LTE (tùy chọn): Quectel EC20 LTE module.

- Micro SIM Slot.

- Internal 4G Antenna + External 4G Sticker Antenna.

\subsubsection{Dragino LoRa Shield.}

Là bộ thu phát LoRa từ xa dựa trên thư viện mã nguồn mở. Lora Shield cho phép người dùng gửi dữ liệu và đạt phạm vi từ $10 \div 15 \mathrm{~km}$. Cung cấp phổ trải rộng và miễn nhiễm cao của hãng Dragino. LoRa Shield được thiết kế theo tiêu chuẩn công nghiệp, nhỏ gọn, dễ dàng lấy tín hiệu về PLC, máy tính, vi xử lý, máy tính nhúng,... (http://dragino. com/; http://bkaii.com.vn/ (Hình 3).

\subsubsection{Cảm biến DHT11}

DHT11 là loại cảm biến số, được dùng để đo nhiệt độ và độ ẩm không khí với độ chính xác cao. Công nghệ chế tạo và thiết kế của DHT11 sensor đảm bảo độ tin cậy cao và ổn định khi hoạt động trong thời gian dài. Cảm biến có chất lượng tốt, mức tiêu thụ điện năng thấp và giá thành thấp (http://bkaii.com.vn/).

- Thông số kỹ thuật: 
- Điện áp hoạt động: 3\%5 V

- Tần số lấy mẫu: $1 \mathrm{~Hz}$

- 4 chân: VCC - Data - NC - GND

\subsubsection{Vi điều khiển Arduino UNO R3}

Vi điều khiển Arduino UNO R3 là kit Arduino UNO thế hệ 3 dùng chip ATmega328P với khả năng lập trình cho các ứng dụng điều khiển phức tạp, do được trang bị cấu hình mạnh cho các loại bộ nhớ ROM, RAM và Flash, các ngõ vào ra digital I/O, trong đó có nhiều ngõ có khả năng xuất tín hiệu PWM (điều chế độ rộng sung), các ngõ đọc tín hiệu analog và các chuẩn giao tiếp đa dạng như UART, SPI, TWI (I2C), (Phạm Quang Huy, Nguyễn Trọng Hiếu, 2014), Hình 4.

Thông số kỹ thuật:

- Điện áp hoạt động: 5V

- Tần số hoạt động: 16 MHz

- Số chân Digital I/O 14 (6 chân hardware PWM).

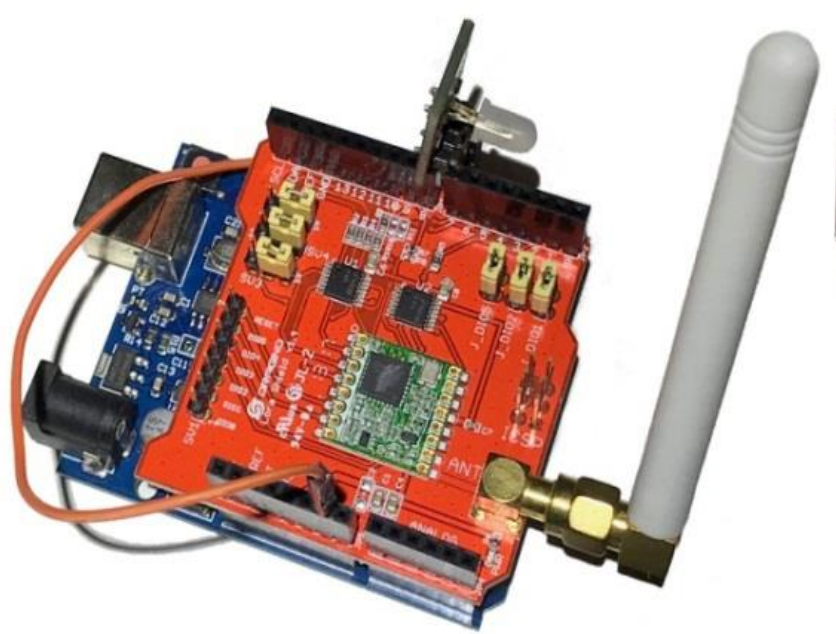

Hình 3. Thiết bị LoRa Shield 433MHz.
- Số chân Analog 6 (độ phân giải 10bit)

\subsection{Thiết kế phần mềm}

\subsubsection{Giao thúc MQTT.}

MQTT (Message Queuing Telemetry Transport) là một giao thức truyền nhận dữ liệu trên nền TCP/IP. MQTT được xây dựng theo mô hình Publish/Subscribe và khái niệm "Topic", giao thức đơn giản và siêu nhẹ thích hợp với các hệ thống nhúng với bộ nhớ và nguồn năng lượng thấp (Hoàng Minh Sơn. 2014).

Hình 5 mô tả nguyên lý hoạt động của giao thức MQTT, các Client (trạm chính) có thể là Publisher (trạm đo và truyền dữ liệu) hoặc Subscriber (giám sát, lưu dữ liệu) hoặc cả hai. Nếu một Publisher gửi dữ liệu vào một Topic (thiết bị gateway) trên MQTT server (được gọi là Broker), ví dụ Topic 2, thì chỉ có các Subscriber Client đã đăng ký vào Topic 2 mới nhận được dữ liệu. Subscriber này sẽ nhận tất cả các dữ liệu được gửi đến Topic 2 từ nhiều Publisher khác.

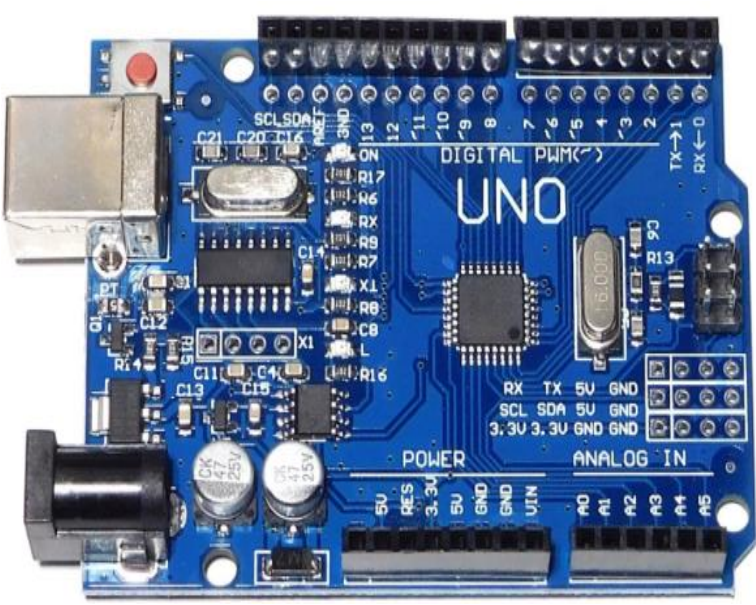

Hình 4. Vi điều khiển Arduino UNO R3.

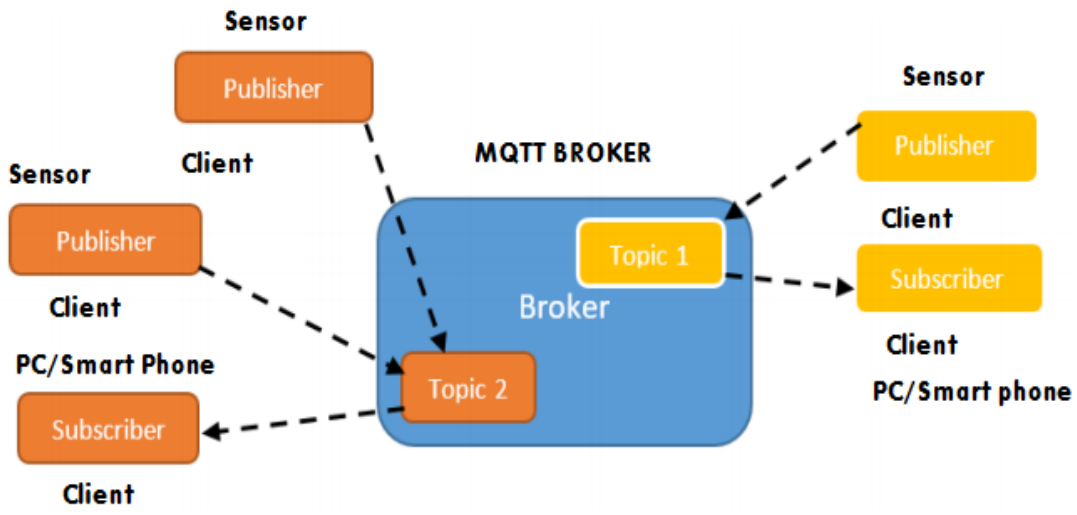

Hình 5. Nguyên lý hoạt động của MQTT. 


\subsubsection{Công cụ Matlab trên Thingspeak Server.}

ThingSpeak ${ }^{\mathrm{TM}}$ là một dịch vụ đám mây nguồn mở cung cấp các ứng dụng IoT (Internet of Things) cho phép người dùng dễ dàng gửi dữ liệu và cung cấp các giao diện đồ họa hiển thị dữ liệu thông qua giao thức HTTP/MQTT qua mạng Internet.

ThingSpeak ${ }^{\mathrm{TM}}$ là một dịch vụ nền tảng phân tích IoT của MathWorks $®$, công cụ MATLAB $\AA$ và Simulink® trên ThingSpeak cho phép tổng hợp, trực quan hóa và phân tích các luồng dữ liệu trực tiếp trên đám mây. ThingSpeak cung cấp các Trend dũ̃ liệu được thực thi bởi mã MATLAB để thực hiện phân tích và xử lý dữ liệu trực tuyến. (http://thinkspeak.com/)

Để sử dụng công cụ Matlab cần phải truy cập vào tài khoản cá nhân như Hình 6.

Sử dụng hàm ThingspeakRead có sẵn trong Matlab để đọc các trường từ các kênh dữ liệu. Hai kênh dữ liệu nhiệt độ và độ ẩm đo trực tuyến với ID1026651, được thực thi bởi mã trong Matlab:
[d,t]=thingSpeakRead (1026651,'DateRange', [datetime ('May 26,2020'),datetime ('May 28, 2020')]);

\subsubsection{Lập trình C code cho Arduino UNO R3.}

Hình 7 là phát triển code được lập trình $\mathrm{C}$ mô tả truyền thông giữa sensor node và vi điều khiển. Gửi dữ liệu lên Thingspeak bằng giao thức MQTT, code lập trình được upload cho Arduino UNO_R3. (Phạm Quang Huy, Nguyễn Trọng Hiếu, 2014)

\section{Kết quả đạt được}

Thiết kế và xây dựng được hệ thống đo giám sát từ xa ứng dụng thiết bị LoRa Gateway có khả năng thu thập giá trị cảm biến nhiệt độ và độ ẩm. Dũ liệu được gửi và lưu trữ trên Web Server. Qua thực nghiệm trên mô hình hệ thống hoạt động theo đúng các yêu cầu đặt ra.

Hình 8 là kết quả hiển thị dữ liệu Field_1 và Field_2 bằng Matlab trên Thingspeak.

Bảng 1 là file cơ sở dữ liệu được tự động ghi lại trong Excel.

\section{MathWorks}

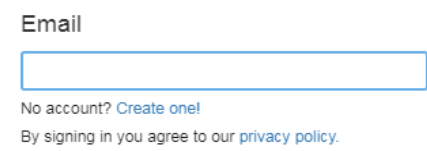

Next

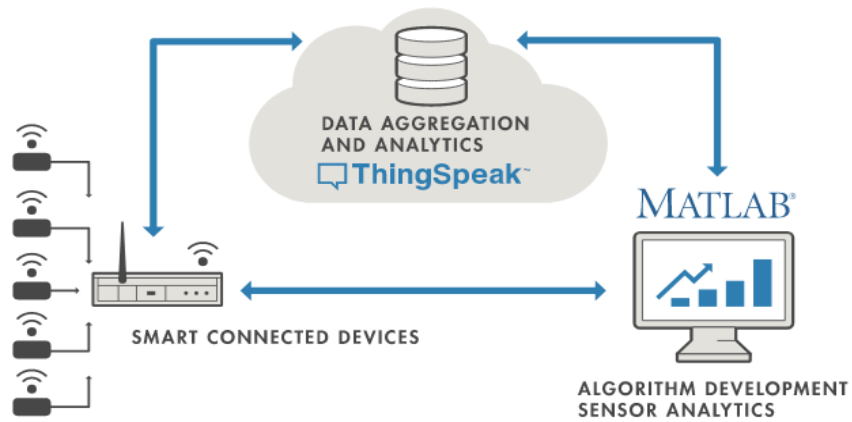

Hình 6. Truy cập vào tài khoản cá nhân để nhận dũ liệu tùr Gateway

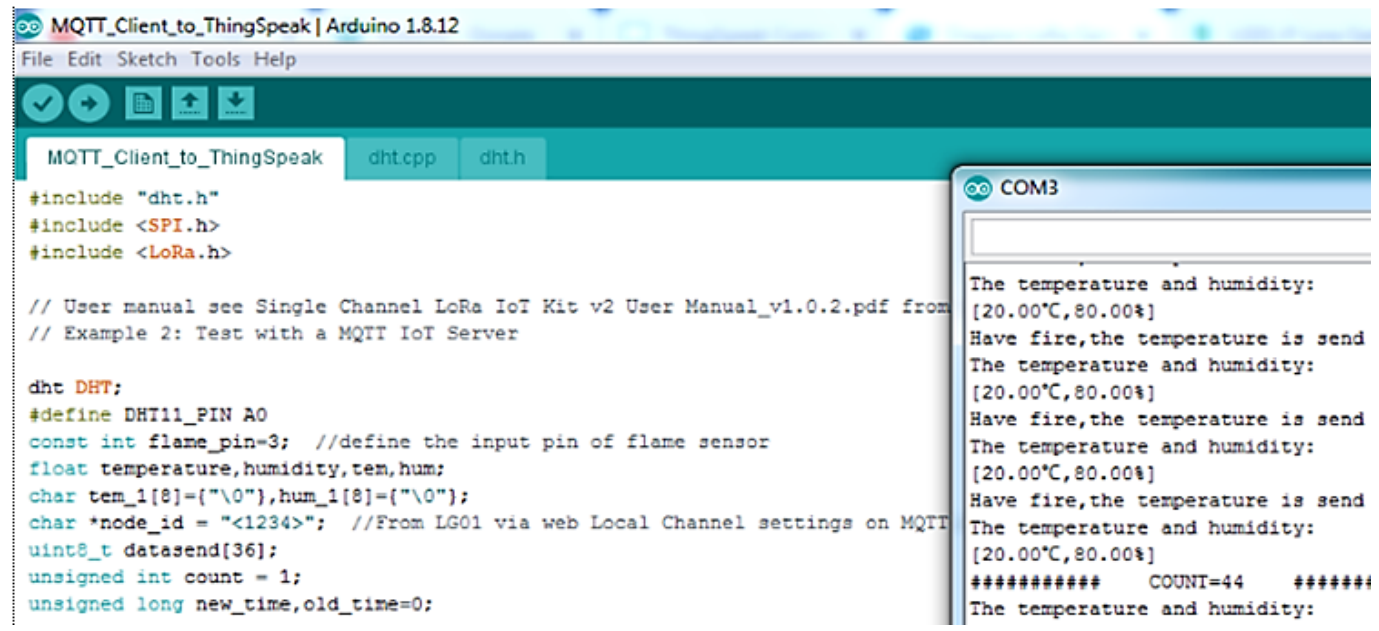

Hình 7. Code cho vi điều khiển Arduino UNO_R3. 


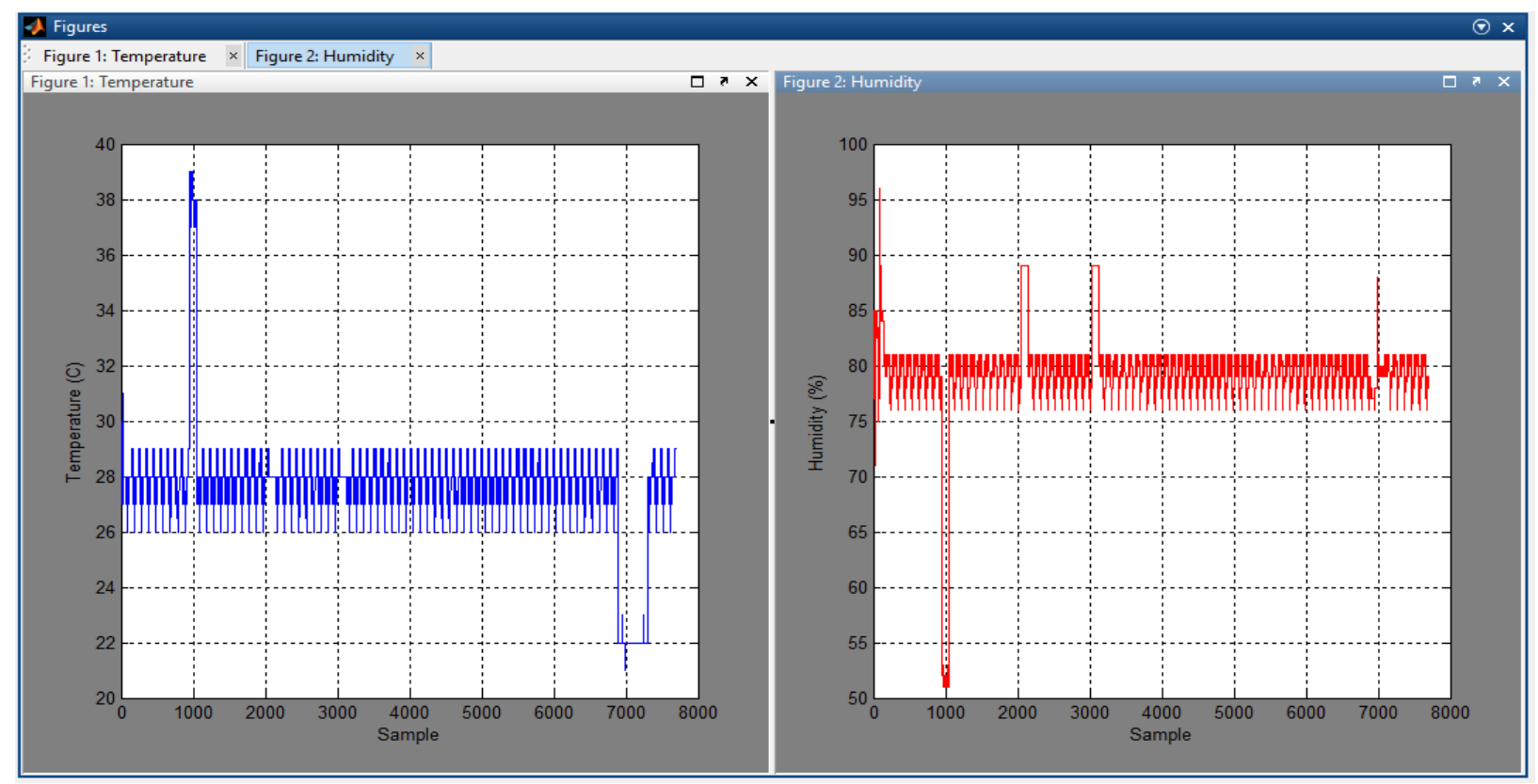

Hình 8. Dũ liệu đo hiển thị dưới dạng biểu đồ Matlab trên Thingspeak.

\begin{tabular}{|c|c|c|c|c|c|c|c|c|}
\hline 4 & A & B & $\mathrm{C}$ & $\mathrm{D}$ & $\mathrm{E}$ & $\mathrm{F}$ & G & $\mathrm{H}$ \\
\hline 37 & 2020-03-26 18:19:06 +07 & 36 & 0 & 0 & & & & MTTQPUBLISH \\
\hline 38 & & & tem_10.0 & hum_10.0 & & & & \\
\hline 39 & $2020-03-26$ 18:20:05 +07 & 37 & 0 & 0 & & & & MTTQPUBLISH \\
\hline 40 & $2020-03-26$ 19:20:38 +07 & 38 & 29 & 85 & & & & MTTQPUBLISH \\
\hline 41 & 2020-03-26 19:21:15+07 & 39 & 29 & 84 & & & & MTTQPUBLISH \\
\hline 42 & $2020-03-26$ 19:22:16 +07 & 40 & 29 & 85 & & & & MTTQPUBLISH \\
\hline 43 & 2020-03-26 19:23:15+07 & 41 & 28 & 84 & & & & MTTQPUBLISH \\
\hline 44 & 2020-03-26 19:24:18+07 & 42 & 28 & 84 & & & & MTTQPUBLISH \\
\hline 45 & 2020-03-26 19:25:18+07 & 43 & 28 & 84 & & & & MTTQPUBLISH \\
\hline 46 & $2020-03-26$ 19:26:21 +07 & 44 & 28 & 84 & & & & MTTQPUBLISH \\
\hline 47 & $2020-03-2619: 27: 27+07$ & 45 & 28 & 84 & & & & MTTQPUBLISH \\
\hline 48 & 2020-03-26 19:28:19+07 & 46 & 28 & 84 & & & & MTTQPUBLISH \\
\hline 49 & $2020-03-26$ 19:33:20 +07 & 47 & 28 & 84 & & & & MTTQPUBLISH \\
\hline 50 & 2020-03-26 19:34:17+07 & 48 & 28 & 84 & & & & MTTQPUBLISH \\
\hline 51 & 2020-03-26 19:36:20 +07 & 49 & 28 & 83 & & & & MTTQPUBLISH \\
\hline 52 & $2020-03-26$ 19:37:16 +07 & 50 & 28 & 81 & & & & MTTQPUBLISH \\
\hline 53 & 2020-03-26 19:38:20 +07 & 51 & 28 & 79 & & & & MTTQPUBLISH \\
\hline 54 & $2020-03-26$ 19:39:21 +07 & 52 & 27 & 77 & & & & MTTQPUBLISH \\
\hline 55 & $2020-03-26$ 19:40:16 +07 & 53 & 27 & 77 & & & & MTTQPUBLISH \\
\hline
\end{tabular}

Hình 9. Hình chụp file cơ sở dũ liệu được lưu tự động trong Worksheet của Excel.

\section{Kết luận và hướng phát triển}

Bài báo trình bày các nội dung nghiên cứu thiết kế, phát triển và thử nghiệm hệ thống giám sát từ xa sử dụng LoRa IoT Gateway LG01-N, công nghệ điện toán đám mây và công nghệ truyền dữ liệu MQTT. Các số liệu đo được giám sát bằng phần mềm Matlab đã được nhúng trong ThingSpeak Server.

Hệ thống đã được tích hợp, lập trình và chạy thử nghiệm cho 1 node sensor trên mô hình giám sát thông số nhiệt độ và độ ẩm. Bước đầu đánh giá cho kết quả khả quan đảm bảo các yêu cầu về kỹ thuật và công nghệ, thu nhận dữ liệu với khoảng cách truyền LoRa tới $10 \mathrm{~km}$. Kết quả nghiên cứu cho phép triển khai hệ thống IoT vào thực tế với các ứng dụng có yêu cầu đo, giám sát từ xa bằng Web Server.

Thiết kế có tính chất thử nghiệm, sử dụng các sensor và thiết bị có giá thành thấp nên cần quan tâm theo dõi đến độ chính xác và ổn định của hệ thống. Một số mẫu đo có sự biến động bất thường (Hình 8) được cho là do nhiễu, tuy nhiên số lượng không nhiều, có thể dùng thuật toán lọc nhiễu nhúng trong Matlab để loại bỏ. Hơn nữa khi triển khai với các ứng dụng ngoài trời thì cũng cần phải chú ý tới các yếu tố ngoại cảnh trong môi trường tác động tới hoạt động của thiết bị. 
Đề xuất tiếp tục đánh giá độ chính xác, tính ổn định và bền vững của hệ thống, mở rộng và phát triển thêm các cảm biến có tính ứng dụng cao trong công nghiệp, nông nghiệp và trong mọi mặt đời sống xã hội với mục tiêu hướng đến một hệ thống làm việc ổn định. Có thể dễ dàng triển khai vào thực tế áp dụng công nghệ LoRa IoT Gateway, đáp ứng kịp thời với sự phát triển rất mạnh mẽ của cuộc cách mạng công nghiệp 4.0.

\section{Tài liệu tham khảo}

Cao Hoàng Tiến, (2015). Nghiên cứu thiết kế hệ thống quan trắc dùng trong nông nghiệp. Hội thảo toàn quốc về CNTT, Trường Đại học Cần Tho, 128-133.

Dự án PAM Air, (2018-2020). Nghiên cứu hệ thống giám sát chất lượng không khí cho các mỏ than lộ thiên Quảng Ninh, Trường Đại học Đông Á (Hàn Quốc) \& Trường Đại học Mỏ - Địa chất; http://humg.edu.vn.

Emara, K. A., Abdeen, M. and Hashem, M., (2009) . A gateway-based framework for transparent interconnection between WSN and IP network. IEEE EUROCON 2009 St.-Petersburg, 17751780.

Hoàng Minh Sơn, (2014), Mạng truyền thông công nghiệp. Nhà xuất bản Khoa học Kỹ thuật, 256tr.

http://bkaii.com.vn/

http://dragino.com/

http://thinkspeak.com/

http://www.arduino.cc/en/Main/ArduinoEther netShield/.

Nasiruddin, M., Rashida Shujaee, (2017). Optimization of A Smart IOT Gateway. International Journal on Recent and Innovation Trends in Computing and Communication. 5 (7), 494 - 501.
Nguyễn Đức Khoát, (2014). A network of wireless temperature sensors based on the zigbee protocol. Conf. Advances in Mining and Tunneling. 21-23/10, Vũng Tàu, Việt Nam, pp431-434.

Nguyễn Đức Ngữ, (2012). Ảnh hưởng của Enso đến các cực trị và lượng mưa ở Việt Nam và khả năng dự báo. Thư viện CRES, Trung tâm KHCN khí tượng thủy văn và môi trường. http://thuviencres.cres.edu.vn/.

Nguyễn Văn Phong, (2018). Ứng dụng IoT trong việc giám sát, cảnh báo mức độ ô nhiễm không khí trong đô thị. http://aita.gov.vn.

Phạm Ngọc Minh, (2015). Phương pháp giám sát và điều khiển các thông số môi trường trên nền tảng điện toán đám mây qua mạng truyền thông không dây WIMAX. Tuyển tập báo cáo Hội nghi toàn quốc lần thứ 3 về Điều khiển và Tự động hóa, 28-29/11, Thái Nguyên, Việt Nam.

Phạm Quang Huy, Nguyễn Trọng Hiếu, (2016). Vi điều khiển và ứng dụng Arduino dành cho người tự học. Nhà xuất bản Đại học Bách Khoa Hà Nội, 407tr.

Pushpa N. Methekar, Girish R. Talmale, (2015). Design and Implementation of IOT Gateway. International Journal of Advance Research, Ideas and Innovations in Technology 3 (2), 295298. ISSN: 2454-132X.

Qian, Z., Ruicong, W., Qi, C., Yan, L., \& Weijun, (2010). IoT Gateway: Bridging Wireless Sensor Networks into Internet of Things. IEEE/IFIP 8th International Conference on the Embedded and Ubiquitous Computing (EUC'2010), 347352.

Vũ Thị Quyên, Phạm Ngọc Minh, Nguyễn Đức Khoát, Ngô Duy Tân, (2018). Thiết kế hệ thống quan sát đối tượng từ xa phục vụ công tác cứu hộ cứu nạn. Tạp chí Khoa học Kỹ thuật Mỏ-Địa chất 59 (1), 1-8. ISSN 1859-1469. 